\title{
A rare case of refeeding syndrome after right hemicolectomy
}

\author{
D. R. Samarathunga, W.A.N.T. Wickramarachchi, Kokulan Thavarasa, S. Sivaganesh \\ University Surgical Unit, National Hospital of Sri Lanka
}

Keywords: Refeeding syndrome; right hemicolectomy; parenteral feeding

\section{Introduction}

Refeeding syndrome (RFS) is characterized by potentially fatal shifts in fluid and electrolytes, that occur in malnourished patients after commencement of enteral or parenteral feeding [1]. It is an underdiagnosed condition that may result in multi-organ failure. We report on a patient who developed RFS after a right hemicolectomy for intestinal obstruction.

\section{Case presentation}

A 53-year-old previously healthy male presented with episodes of abdominal colics, bilious vomiting and progressive anorexia of six months duration. He had been on a low-calorie liquid diet due to his symptoms over the previous two months duration and lost $20 \mathrm{~kg}$ in weight during this period. Past surgical history was unremarkable. He was wasted with a body mass index (BMI) of $15.1 \mathrm{~kg} / \mathrm{m} 2$. The mid-arm circumference was $16.5 \mathrm{~cm}$. The abdomen was scaphoid but otherwise normal except for visible peristalsis. Other systems were normal. Key biochemical parameters from admission to discharge are shown in figure 1.

Oesophago-gastro-duodenoscopy (OGD) showed healed antral ulcers and sequestered bile. Contrast-enhanced CT abdomen revealed an annular growth in the distal jejunum without evidence of loco-regional extension or metastatic disease.

The patient was nutritionally optimized for one week with parenteral nutrition of $30 \mathrm{kcal} / \mathrm{kg} /$ day and intravenous thiamine supplementation before surgery. Laparotomy revealed an annular growth in the proximal jejunum and a primary growth at the ileocaecal junction with metastatic omental deposits. A right hemicolectomy with ileocolic anastomosis and a jejunal resection with primary side to side anastomosis was done. Postoperatively parenteral nutrition

Correspondence: D. R. Samarathunga

E-mail: dishanrandika@live.com

(iD) https://orcid.org/0000-0002-4407-7021

Received: 18-02-2021 Accepted: 14-11-2021 DOI: http://doi.org/10.4038/sljs.v39i3.8804 was continued at $30 \mathrm{kcal} / \mathrm{kg} /$ day and oral intake was introduced incrementally.

On the 2nd postoperative day, he developed abdominal distension with sluggish bowel sounds. Paralytic ileus was diagnosed. Over the following two days, he demonstrated extreme fatigue, muscle weakness, resting tremor and paraesthesia in his extremities. In addition, he became disoriented and displayed episodes of aggressive behaviour. This was followed by hypotension and oliguria that did not respond to fluid resuscitation and required noradrenaline infusion. Echocardiography showed an ejection fraction of $50 \%$ without segmental hypokinesia. He developed bilateral pleural effusions, ascites, bilateral leg oedema and finally coffee ground vomiting. Ultrasonography excluded deep vein and portal vein thrombosis. He was afebrile and clinical features and inflammatory markers were not in favour of sepsis.

Biochemical assays demonstrated a progressive reduction in serum ionized calcium, magnesium and phosphate levels after commencing therapeutic feeding with the lowest values noted between the 3 rd and 5 th postoperative day.

Thrombocytopenia was observed with values as low as $10,000 / \mathrm{mm} 3$ that coincided with the upper gastrointestinal bleeding.

The evolving clinical presentation resulted in the lack of a diagnosis until the 5 th postoperative day when refeeding syndrome was diagnosed. The caloric intake was immediately reduced to $10 \mathrm{kcal} / \mathrm{kg} /$ day over the next 4 days and gradually increased to $15 \mathrm{kcal} / \mathrm{kg} /$ day. Enteral feeding was established by the 7 th postoperative day after the paralytic ileus settled. Intravenous calcium gluconate, magnesium sulphate, thiamine and oral phosphate supplementation were initiated. These resulted in the gradual resolution of clinical parameters and biochemical derangements. He was discharged on the 14 th postoperative day on a solid high protein, normal calorie diet.

Histology revealed an adenocarcinoma of the caecum with likely small bowel and omental metastatic disease. He was referred to the oncology unit for adjuvant systemic therapy. 

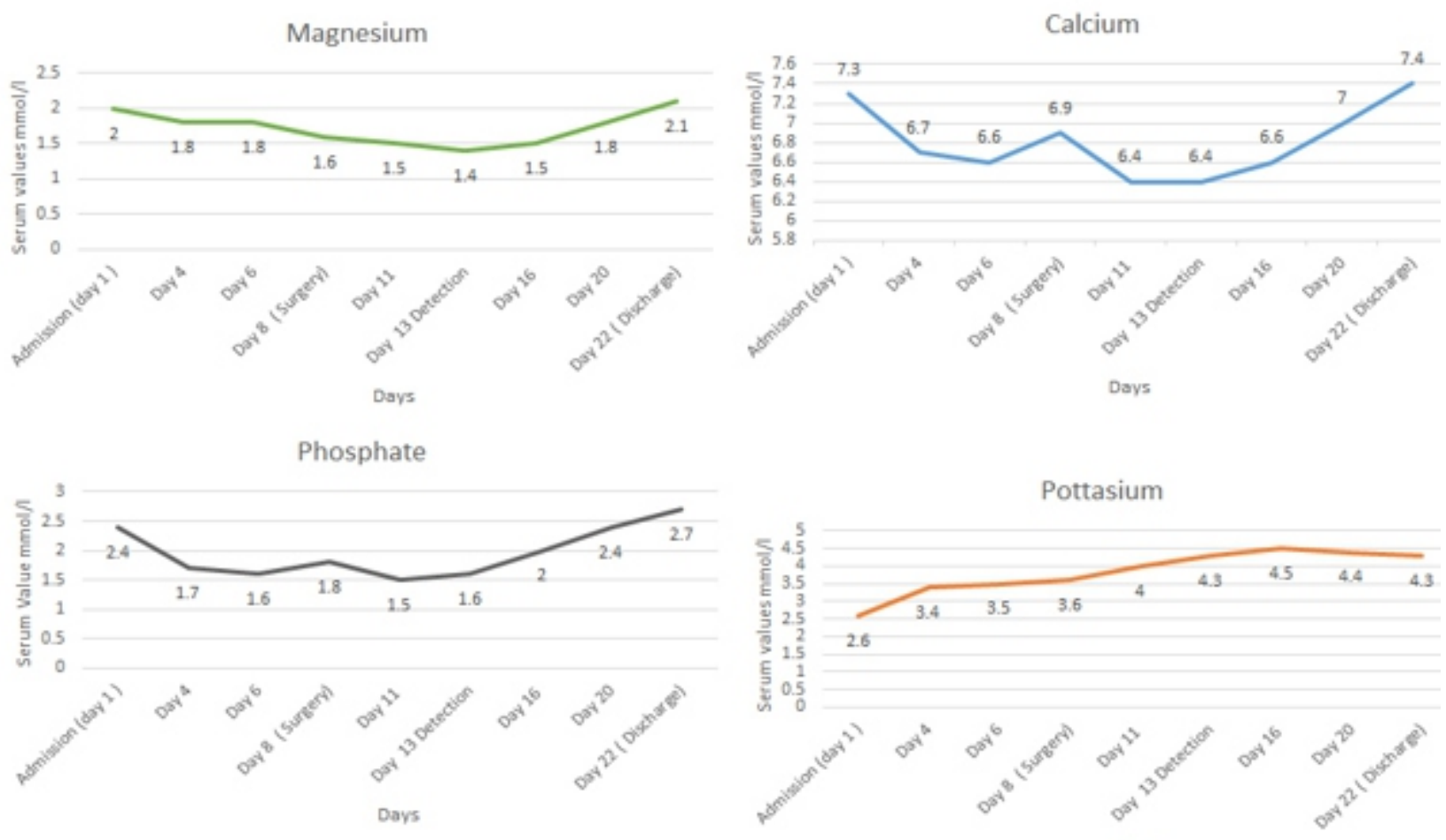

Figure 1. Demonstrate the electrolyte shifts of this patient. From the day of admission serum electrolyte levels were gradually decreasing until identification of RFS (Day 13). Symptoms appear around day 11 . Lowest values are seen around day 13. With reduction in calorie intake and intravenous electrolyte supplementation gradual correction of electrolytes is seen

He is on a normal diet and engaged in daily activities while on chemotherapy.

\section{Discussion}

RFS is a potentially fatal condition that occurs due to metabolic and hormonal shifts in high-risk patients after the commencement of supplemental feeding. As in this patient, early clinical features are often non-specific [2] leading to missed and late diagnoses and treatment [3].

Early identification of high-risk patients and a high index of suspicion and are important in the management of RFS. The National Institute for Health and Clinical Excellence (NICE2006) guidelines to identify high-risk patients are indicated in Table 1. Based on these guidelines the patient was at high risk with a BMI less than $16 \mathrm{~kg} / \mathrm{m} 2$, weight loss of $37.7 \%$ over six months, minimal nutrition intake for more than 10 days and initial low potassium and phosphate levels.

RFS is characterised by fluid retention, hypocalcaemia, hypomagnesaemia, hypokalaemia and hypophosphataemia. Starvation leads to chronic depletion of electrolytes, fluid and energy followed by glycogenolysis and later gluconeogenesis. During refeeding, there is a shift to carbohydrate metabolism and ingested glucose induces insulin secretion. This provokes glucose uptake and increased cellular metabolism with intracellular uptake of calcium, potassium, magnesium and phosphate resulting in further reduction in serum levels. Furthermore, the shift to carbohydrate metabolism reduces sodium and water excretion resulting in extracellular fluid accumulation and fluid overload [3].

In retrospect, the clinical course of this patient is explained by the mechanism described above. The hypokalaemia resulted in reduced cardiac contractility, hypotension, fatigue,

Table 1. National Institute for Health and Clinical Excellence guidelines for identifying patients at high risk of refeeding problems [5].

\begin{tabular}{|c|}
\hline One or more of the following: \\
\hline Body mass index $(\mathrm{kg} / \mathrm{m} 2)<16$ \\
\hline $\begin{array}{l}\text { Unintentional weight loss }>15 \% \text { in the past three to six } \\
\text { months }\end{array}$ \\
\hline Little or no nutritional intake for $>10$ days \\
\hline $\begin{array}{l}\text { Low levels of potassium, phosphate, or magnesium } \\
\text { before feeding }\end{array}$ \\
\hline Two or more of the following: \\
\hline Body mass index $<18.5$ \\
\hline $\begin{array}{l}\text { Unintentional weight loss }>10 \% \text { in the past three to six } \\
\text { months }\end{array}$ \\
\hline Little or no nutritional intake for $>5$ days \\
\hline $\begin{array}{l}\text { History of alcohol misuse or drugs, including insulin, } \\
\text { chemotherapy, antacids, or diuretics }\end{array}$ \\
\hline
\end{tabular}


weakness and ileus. The hypophosphataemia and hypomagnesaemia caused altered mental status, seizures and paraesthesia and finally thrombocytopenia. His altered sensorium was likely further compounded by thiamine deficiency.

The management of RFS includes nutritional assessment and identification of high-risk patients, close monitoring and replacement of electrolytes, fluids and calorie intake. The feeding regimen should be individualized to each high-risk patient. Feeding is started at no more than $50 \%$ of the daily requirement, usually at $10 \mathrm{kcal} / \mathrm{kg} / \mathrm{day}$ for high-risk patients and gradually increased [1]. Before refeeding, phosphate, magnesium and potassium assays must be done. Early correction of vitamin deficiency, especially thiamine, is done before the commencement of feeding and continued for at least 10 days [1].

Correction of hypophosphataemia and hypomagnesaemia is necessary if serum levels are $<0.30 \mathrm{mmol} / \mathrm{L}$ or $<0.50 \mathrm{mmol} / 1$ respectively or if symptomatic as was observed in this case [4]. The correction of electrolytes can be done concurrently with feeding. Monitoring for arrhythmias, fluid overload and frequent monitoring of serum and urinary electrolytes are mandatory [1].
All authors disclose no conflict of interest. The study was conducted in accordance with the ethical standards of the relevant institutional or national ethics committee and the Helsinki Declaration of 1975, as revised in 2000 .

\section{References}

1. Mehanna, H.M., J. Moledina, and J. Travis, Refeeding syndrome: what it is, and how to prevent and treat it. BMJ (Clinical research ed.), 2008. 336(7659): p. 1495-1498. https://doi.org/10.1136/bmj.a301

2. Hearing, S.D., Refeeding syndrome. BMJ (Clinical research ed.), 2004.328(7445): p. 908-909.

https://doi.org/10.1136/bmj.328.7445.908

3. Hammami, S., et al., Refeeding syndrome in adults with celiac crisis: a case report. Journal of medical case reports, 2018. 12(1): p. 22-22. https://doi.org/10.1186/s13256-018-1566-6

4. Crook, M.A., V. Hally, and J.V. Panteli, The importance of the refeeding syndrome. Nutrition, 2001. 17(7-8): p. 632-7. https://doi.org/10.1016/S0899-9007(01)00542-1

5. National Institute for Health and Clinical Excellence. Nutrition support in adults. Clinical guideline CG32. 2006. Available: www.nice.org.uk/page.aspx $? \mathrm{o}=\operatorname{cg} 032$

\section{Learning Points:}

- RFS is a potentially fatal condition

- Clinical assessment and biochemical screening will identify those at high risk of RFS

- Measured caloric supplementation with electrolyte and vitamin replacement will minimise occurrence and sequelae of RFS 